\title{
DERIVATIONS AND EMBEDDINGS OF A FIELD IN ITS POWER SERIES RING
}

\section{NICKOLAS HEEREMA}

I. Introduction. Let $F$ be a field and $F[[x]]$ its power series ring in the single indeterminate $x$. It is well known that, if $F$ is a perfect field $F$ is embedded in $F[[x]]$ in only one way. In the literature [2] this fact is obtained as a consequence of the uniqueness of the multiplicative representatives of $F$ as residue field. This unique embedding property can also be very easily obtained as a consequence of the fact that $F$ is perfect only if it has no nontrivial derivations by a technique used by the author in a slightly different context [1].

The object of this paper is to show how in case $F$ has characteristic zero, the various ways in which $F$ is embedded in $F[[x]]$ can be determined constructively and biuniquely from countable sequences of derivations on $F$. We consider only those embeddings $F^{\prime}$ of $F$ with the property that $F^{\prime}$ maps onto $F$ as residue field when the maximal ideal in $F[[x]]$ is removed. Implications regarding the group of automorphisms of $F[[x]]$ are also discussed.

The author noticed after submitting the first three sections of this paper that the proofs contained therein can be used with practically no modification to obtain analogous results for the case in which $F[[x]]$ is replaced by the power series ring $F\left[\left[x_{1}, \cdots, x_{n}\right]\right]$ in $n$ indeterminates. These results with appropriate definitions are given in $\S I V$.

II. Embeddings and derivations. Let $F$ be isomorphic to $F^{\prime}$ $C F[[x]]$ by the mapping $a \rightarrow \sum a_{i} x^{i}$. (Throughout this paper $F$ will be identified with the set of power series of the form $a+0 x+\cdots$ $\left.+0 x^{n}+\cdots\right)$ The mappings $\bar{\pi}_{i}$ on $F(i=1,2, \cdots)$ given by $\bar{\pi}_{i}\left(a_{0}\right)$ $=a_{i}$ satisfy the two conditions

$$
\begin{aligned}
\bar{\pi}_{n}(a+b) & =\bar{\pi}_{n}(a)+\bar{\pi}_{n}(b), \\
\bar{\pi}_{n}(a b) & =\sum_{i=0}^{n} \bar{\pi}_{i}(a) \bar{\pi}_{n-i}(b)
\end{aligned}
$$

where $\bar{\pi}_{0}(a)=a$.

Conversely, given a sequence $\left\{\bar{\pi}_{i}\right\}_{1}^{\infty}$ of mappings on $F$ satisfying conditions (1) and (2) and an automorphism $a \rightarrow a_{0}$ on $F$ the mapping $a \rightarrow a_{0}+\bar{\pi}_{1}\left(a_{0}\right) x+\cdots+\bar{\pi}_{i}\left(a_{0}\right) x^{i}+\cdots$ is an isomorphism of $F$ into $F[[x]]$. A sequence $\left\{\bar{\pi}_{i}\right\}_{1}^{\infty}$ of mappings on $F$ satisfying conditions

Presented to the Society, April 17, 1959; received by the editors April 20, 1959 and, in revised form, May 24, 1959. 
(1) and (2) will be called an embedding sequence.

In order to establish the connection between embedding sequences and sequences of derivations some conventions and a lemma are required. Let $(r, n)$ represent a partition of the integer $n$ into $r$ positive summands. Let $(r, n)$, denote a partition $(s, m)$ whose summands are taken from $(r, n)$ and let $(r, n)_{s}^{*}$ denote the complimentary subpartition composed of the remaining indices in $(r, n)$. Thus $(r, n)_{1}$, for example, would be some one of the indices in $(r, n)$. Let $\left\{\eta_{i}\right\}$ be a sequence of mappings of $F$ into $F$ and let $[\eta]_{(r, n)}$ represent the sum of all formally distinct products of the $r$ mappings $\eta_{i}$ for $i \in(r, n)$ (i.e., $[\eta]_{1,1,1}=\eta_{1}^{3}$ and $\left.[\eta]_{1,1,2}=\eta_{1}^{2} \eta_{2}+\eta_{1} \eta_{2} \eta_{1}+\eta_{2} \eta_{1}^{2}\right)$. The symbol $[\eta]_{(r, n)_{0}}$ will represent the identity map.

Leмma. If $\left\{\pi_{i}\right\}_{1}^{\infty}$ is a sequence of derivations of a commutative ring $R$ into a commutative ring $S$ containing $R$ then

$$
[\pi]_{(r, n)}(a b)=\sum_{(r, n)_{s}} C_{r, s}[\pi]_{(r, n)_{\triangleleft}}(a)[\pi]_{(r, n)_{s}^{*}(b) .}
$$

Proof. The lemma holds for $r=1$ for then $[\pi]_{(r, n)}=\pi_{n}$ and the only subpartitions of $(r, n)$ are $(r, n)_{0}$ and $(r, n)_{0}^{*}$. Proceeding by induction on the number $r$ of indices in $(r, n)$ we obtain

$$
\begin{aligned}
& {[\pi]_{(r, n)}(a b)=\sum_{(r, n)_{1}=j}[\pi]_{(r, n)_{1}^{*}\left(\pi_{j}(a b)\right)}} \\
& =\sum_{(r, n)_{1}=j}[\pi]_{(r, n)_{1}^{*}}\left(a \pi_{j}(b)+b \pi_{j}(a)\right) \\
& =\sum_{(r, n)_{1}=j} \sum_{\left((r, n)_{i}^{*}\right)_{i}} C_{r-1, i}\left\{[\pi]_{\left.((r, n))_{i}^{*}\right)_{i}^{*}(a)}[\pi]_{\left((r, n)_{1}^{*}\right)_{i}}\left(\pi_{j}(b)\right)\right.
\end{aligned}
$$

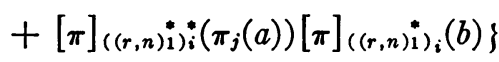

To establish that this last expression is the same as the right side of (3) we note they have the same set of terms and that a particular term of $[\pi]_{(r, n) i}(a)[\pi]_{(r, n)_{i}}(b)$ appears twice in expression (4) with coefficients $C_{r-1, i}$ and $C_{r-1, i-1}$. The lemma follows then from the observation that

$$
C_{r, i}=C_{r-1, i}+C_{r-1, i-1}
$$

If $R$ has prime characteristic $p$ we have the following generalization of the well known fact that if $\pi$ is a derivation on $R$ into $S$ so is $\pi^{p}$.

CoROllary 1. $[\pi]_{(p, n)}$ is a derivation.

Henceforth, $F$ is assumed to have characteristic zero. 
THEOREM 1. The sequence of mappings $\left\{\bar{\pi}_{i}\right\}$ defined by relations (5) below in terms of a sequence of derivations $\left\{\pi_{i}\right\}$ on $F$ is an embedding sequence on $F$.

$$
\bar{\pi}_{n}=\sum_{(r, n)} \frac{[\pi]_{(r, n)}}{r !}, \quad n=1,2, \cdots
$$

Proof. The mapping $\bar{\pi}_{n}$ is clearly an additive homomorphism. In order to verify relation (2) we use the lemma on both sides of (2) as follows.

Also,

$$
\begin{aligned}
\bar{\pi}_{n}(a b) & =\sum_{(r, n)} \frac{[\pi]_{(r, n)}}{r !}(a b), \\
& =\sum_{(r, n)} \sum_{(r, n)_{i}} \frac{[\pi]_{(r, n)_{i}^{*}}(a)}{(r-i) !} \frac{[\pi]_{(r, n)_{i}}(b)}{i !}
\end{aligned}
$$

$$
\sum_{i=0}^{n} \bar{\pi}_{i}(a) \bar{\pi}_{n-i}(b)=\sum_{i=0}^{n}\left\{\sum_{(r, i)} \frac{[\pi]_{(r, i)}(a)}{r !} \sum_{(s, n-i)} \frac{[\pi]_{(s, n-i)}(b)}{s !}\right\},
$$

which is seen to be the same as (6) above.

TheOREM 2. The mappings $\eta_{n}$ defined by relation (7) below, in terms of an embedding sequence $\left\{\bar{\eta}_{i}\right\}$ on $F$, are derivations.

$$
\eta_{n}=\sum_{(r, n)} \frac{(-1)^{r+1}}{r}[\bar{\eta}]_{(r, n)}, \quad n=1,2, \cdots
$$

Proof. It is clear that $\eta_{n}$ is an additive homomorphism on $F$. To show that $\eta_{n}(a b)=a \eta_{n}(b)+b \eta_{n}(a)$ we note that

$$
\begin{aligned}
& \eta_{n}(a b)=\sum_{(r, n)} \frac{(-1)^{r+1}}{r}[\bar{\eta}]_{(r, n)}(a b) \\
& =\sum_{[r, n]=i_{1}, \cdots, i_{r}} \frac{(-1)^{r+1}}{r} \sum_{j_{1}, \ldots, j_{r} ; 0 \leq j_{t} \leq i_{t} ; t=1, \ldots, r} \bar{\eta}_{j_{1}} \cdots \\
& \bar{\eta}_{j_{r}}(a) \bar{\eta}_{i_{1}-j_{1}} \cdots \bar{\eta}_{i_{r}-j_{r}}(b),
\end{aligned}
$$

where the left summation is taken over all ordered partitions $[r, n]$ of $n$ into positive summands.

The coefficient of a particular term $\eta_{i_{1}} \cdots \eta_{i_{s}}(a) \eta_{j_{1}} \cdots \eta_{j_{m}}(b)$ of (8), assuming $m \geqq s$, is found to be

$$
\sum_{j=0}^{s} \frac{(-1)^{m+s-j+1}}{m+s-j}\left(C_{m+s-j, m}\right)\left(C_{m, j}\right) .
$$


We need to show that (9) vanishes for $s>0$ and equals $(-1)^{m+1} / m$ if $s=0$, the latter being obvious. It will follow then by symmetry that the mapping $\eta_{n}$ is a derivation. To this end we note that (9) can be rewritten as

$$
\frac{(-1)^{m-s+1}}{m} \sum_{j=0}^{s}(-1)^{j}\left(C_{m-1+s-j, s-j}\right)\left(C_{m, j}\right) .
$$

However,

$$
\sum_{j=0}^{s}(-1)^{i}\left(C_{m-1+8-j, 8-j}\right)\left(C_{m, j}\right)
$$

is the coefficient of $x^{8}$ in the power series expansion of $(1-x)^{-m}(1-x)^{m}$ and, hence, is zero for $s>0$.

Let $\varepsilon\left\{\pi_{i}\right\}$ denote the embedding sequence determined by $\left\{\pi_{i}\right\}$ using relation (5) and let $D\left\{\bar{\pi}_{i}\right\}$ be the sequence of derivations determined by the embedding sequence $\left\{\bar{\pi}_{i}\right\}$ using (7).

ThEOREM 3. $E D\left\{\bar{\pi}_{i}\right\}=\left\{\bar{\pi}_{i}\right\}$.

Proof. Let $\left\{\pi_{i}\right\}=D\left\{\bar{\pi}_{i}\right\}$ and $\left\{\bar{\eta}_{i}\right\}=\varepsilon\left\{\pi_{i}\right\}$. Then

$$
\begin{aligned}
\bar{\eta}_{n}= & \sum_{[r, n]=j_{1}, \cdots, j_{r}} \frac{1}{r !} \pi_{j_{1}} \cdots \pi_{j_{r}}, \\
= & \sum_{[r, n]=j_{1}, \cdots, j_{r}} \frac{1}{r !}\left\{\left[\sum_{\left(s_{1}, j_{1}\right)} \frac{(-1)^{s_{1}+1}}{s_{1}}[\bar{\pi}]_{\left(s_{1}, j_{1}\right)}\right] \cdots\right. \\
& {\left.\left[\sum_{\left(s_{r}, j_{r}\right)} \frac{(-1)^{s_{r}+1}}{s_{r}}[\bar{\pi}]_{\left(s_{r}, j_{r}\right)}\right]\right\} . }
\end{aligned}
$$

The coefficient of a particular term $\bar{\pi}_{k_{1}} \cdots \bar{\pi}_{k_{r}}$ of (10) is seen to be equal to the following

$$
(-1)^{r} \sum_{[s, r]=n_{1}, \cdots, n_{8}} \frac{(-1)^{s}}{s !} \frac{1}{n_{1} n_{2} \cdots n_{s}} .
$$

However

$$
\sum_{[8, r]=n_{1}, \cdots, n_{8}} \frac{(-1)^{s}}{s !} \frac{1}{n_{1} n_{2} \cdots n_{8}}
$$

is the coefficient of $x^{r}$ in the power series expansion of

$$
e^{\log |1-x|}=\sum_{s=0}^{\infty} \frac{1}{s !} \log ^{s}|1-x| \text {. }
$$


Thus (11) vanishes for $r>1$ and is equal to one for $r=1 .{ }^{1}$ From this it follows that $\bar{\eta}_{n}=\bar{\pi}_{n}$ which proves Theorem 3 .

Let $F$ be a field having characteristic zero and let $F_{\left\{\pi_{i}\right\}}$ denote the subfield of $F[[x]]$ of all elements of the form $a+\bar{\pi}_{1}(a) x+\ldots$ $+\bar{\pi}_{n}(a) x^{n}+\cdots$ where $\left\{\bar{\pi}_{i}\right\}=\varepsilon\left\{\pi_{i}\right\}$ and $\left\{\pi_{i}\right\}$ is a sequence of derivations on $F$. We have proved the following.

THEOREM 4. Let $F$ be isomorphic to $F^{\prime} \subset F[[x]]$ under the mapping $a \rightarrow \sum a_{i} x^{i}$. Then the mappings $\left\{\bar{\pi}_{i}\right\}$ on $F$ given by $\bar{\pi}_{i}\left(a_{0}\right)=a_{i}$ form an

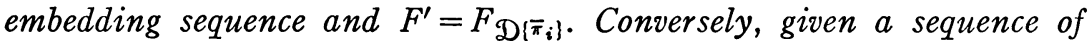
derivations $\left\{\pi_{i}\right\}$ on $F, F$ is isomorphic to $F_{\left\{\pi_{i}\right\}}$ under the mapping $a \rightarrow a+\bar{\pi}_{1}(a) x+\cdots+\bar{\pi}_{n}(a) x^{n}+\cdots$ where $\left\{\bar{\pi}_{i}\right\}=\varepsilon\left\{\pi_{i}\right\}$. The correspondence between sequences of derivations on $F$ and embeddings of $F$ in $F[[x]]$ is biunique.

Let $\pi_{1}$ be nontrivial and let $\pi_{i}, i=2, \cdots$, be the zero map. Then if some power of $\pi_{1}$ is trivial $F_{\left\{\pi_{i}\right\}}$ is in the polynomial ring $F[x]$. Since every field is uniquely embedded in its polynomial ring we have

CoROllary 2. If $\pi$ is a nontrivial derivation on the field $F$ of characteristic zero then $\pi^{k}$ is nontrivial for all positive integers $k$.

III. An implication. Let $\alpha$ be an automorphism of $F[[x]]$. The mapping $\alpha$ is uniquely determined by three of its properties. First, $\alpha$ maps $F$ onto some $F_{\left\{\pi_{i}\right\}}$ as follows $\alpha(a)=\sum \bar{\pi}_{i}\left(a_{0}\right) x^{i}$ where $a \rightarrow a_{0}$ is an automorphism $\alpha^{*}$ on $F$ and $\left\{\bar{\pi}_{i}\right\}=\varepsilon\left\{\pi_{i}\right\}$. Also $\alpha(x)=\sum_{i=1}^{\infty} c_{i} x^{i}$ for $c_{1} \neq 0$. Thus $\alpha$ is determined by $\left(\alpha^{*},\left\{\pi_{i}\right\},\left\{c_{i}\right\}\right)$. Conversely, such a triple determines an automorphism of $F[[x]]$ and the correspondence is biunique.

Let $G$ be the group of automorphisms of $F[[x]]$ and let $G_{1}$ be the subgroup of those automorphisms $\alpha$ such that $\alpha(x)=x$ and $\alpha^{*}$ is the identity. The group $G_{1}$ is isomorphic to a group $H_{1}$ whose elements are sequences of derivations the group operation being defined in terms of symmetric products of the derivations involved. The group operation on $H_{1}$ can be used in turn to define the product of triples $\left(\alpha^{*},\left\{\pi_{i}\right\},\left\{c_{i}\right\}\right) \cdot\left(\beta^{*},\left\{\eta_{i}\right\},\left\{b_{i}\right\}\right)$ so as to obtain a group $H$ isomorphic to $G$. This subject will, it is hoped, be treated in another paper.

IV. Embeddings of $F$ in $F\left[\left[x_{1}, \cdots, x_{n}\right]\right]$. With the following conventions the results of $\S$ II can be extended to apply to the power series ring $F\left[\left[x_{1}, \cdots, x_{n}\right]\right]$ in $n$ indeterminates.

Let $I$ denote an $n$-tuple of non-negative integers $m_{1}, \cdots, m_{n}$. Let $g^{*}$ represent the set of all $I$ and $\mathscr{g}$ the set of all $I$ with the exception

${ }^{1}$ The author is indebted to Dr. Marion F. Tinsley for the above proofs that (11) and (9) vanish for appropriate values of $s$ and $r$. 
of the zero $n$-tuple $0, \cdots, 0$. For $I$ in $g$ the symbol $(r, I)$ will represent a set of $r n$-tuples $I_{1}, \cdots, I_{r}$ in $g$ such that $I_{1}+\cdots+I_{r}=I$ where addition is defined component-wise. Thus $(r, I)$ is a partition of $I$ into $r$ nonzero summands. Let $(r, I)_{s}$ and $(r, I)_{s}^{*}$ denote a subset of $s n$-tuples in $(r, I)$ and its complimentary set respectively.

The elements of $F\left[\left[x_{1}, \cdots, x_{n}\right]\right]$ have the form $\sum_{I \in \mathcal{g}^{*}} a_{I} x^{I}$ where $x^{I}$ represents $x_{1}^{i_{1}} \cdots x_{n}^{i_{n}}$ for $I=i_{1}, \cdots, i_{n}$. As in $\S I I$ an isomorphism of $F$ onto $F^{\prime} \subset F\left[\left[x_{1}, \cdots, x_{n}\right]\right]$ determines and is determined by an automorphism on $F$ and an embedding sequence $\left\{\bar{\pi}_{I}\right\}_{I \in \mathcal{G}}$ which satisfies conditions $\left(1^{\prime}\right)$ and $\left(2^{\prime}\right)$ below.

$$
\begin{aligned}
\bar{\pi}_{I}(a+b) & =\bar{\pi}_{I}(a)+\bar{\pi}_{I}(b), \\
\bar{\pi}_{I}(a b) & =\sum_{J \leq I} \bar{\pi}_{J}(a) \bar{\pi}_{I-J}(b) .
\end{aligned}
$$

Here, $J \leqq I$ if and only if each component of $J$ is less than or equal to the corresponding component of $I$ and $\bar{\pi}_{0,} \ldots, 0$ is the identity mapping.

Given a sequence of mappings $\left\{\eta_{I}\right\}_{g}$ on $F$ into $F$ we define $[\eta]_{(r, I)}$ in a manner analogous to the definition of $[\eta]_{(r, n)}$.

The proofs of the following results differ from the proofs of the corresponding results in $\S I I$ only in that $(r, n)$ is replaced by $(r, I)$ with corresponding changes in the sets over which sums are taken.

Lemma. If $\left\{\pi_{I}\right\}_{g}$ is a sequence of derivations of a commutative ring $R$ into a commutative ring $S$ containing $R$ then

$$
[\pi]_{(r, I)}(a b)=\sum_{(r, I)} C_{r, s}[\pi]_{(r, I)_{0}}(a)[\pi]_{(r, I)^{*}}^{*}(b) .
$$

Corollary $1^{\prime}$. If $R$ has prime characteristic $p$ then $[\pi]_{(p, I)}$ is a derivation.

We assume again that $F$ has characteristic zero.

THEOREM $1^{\prime}$. The sequence of mappings $\left\{\bar{\pi}_{I}\right\}_{g}$ defined by relations $\left(5^{\prime}\right)$ below, in terms of a sequence of derivations $\left\{\pi_{I}\right\} g$ defined on $F$ into $F$, is an embedding sequence on $F$.

$$
\bar{\pi}_{I}=\sum_{(r, I)} \frac{[\pi]_{(r, I)}}{r !}
$$

TheOREM $2^{\prime}$. The mappings $\eta_{I}$ defined by relation $\left(7^{\prime}\right)$ below, in terms of an embedding sequence $\left\{\bar{\eta}_{I}\right\} g$ on $F$, are derivations.

$$
\eta_{I}=\sum_{(r, I)} \frac{(-1)^{r+1}[\bar{\eta}]_{(r, I)}}{r}, \quad I \in g .
$$


Defining $\mathcal{E}^{\prime}\left\{\pi_{I}\right\}_{\mathscr{S}}$ and $\mathscr{D}^{\prime}\left\{\bar{\pi}_{I}\right\}_{\mathscr{g}}$ respectively to be the embedding sequence, and the sequence of derivations given by relations $\left(5^{\prime}\right)$ and $\left(7^{\prime}\right)$ we have

ThEOREM $3^{\prime} . \mathcal{E}^{\prime} \mathfrak{D}^{\prime}\left\{\bar{\pi}_{I}\right\} g=\left\{\bar{\pi}_{I}\right\} g$.

We define $F_{\left\{\pi_{I}\right\}}$, where $\left\{\pi_{I}\right\}_{g}$ is a sequence of derivations on $F$, to be the set of elements $\sum g^{*} \bar{\pi}_{I}(a) x^{I}$, where $\left\{\bar{\pi}_{I}\right\}_{g}=\mathcal{E}^{\prime}\left\{\pi_{I}\right\} g$.

Theorem 4. Let $F$ be isomorphic to $F^{\prime} \subset F\left[\left[x_{1}, \cdots, x_{n}\right]\right]$ under the mapping $a \rightarrow \sum g^{*} a_{I} x^{I}$. Then, the sequence of mappings $\left\{\bar{\pi}_{I}\right\}$ given by $\bar{\pi}_{I}\left(a_{0}, \ldots, 0\right)=a_{I}$ form an embedding sequence and $F^{\prime}=F_{D^{\prime}}\left\{\bar{\pi}_{I}\right\}$. Conversely, given a sequence of derivations $\left\{\pi_{I}\right\}_{g}$ on $F, F$ is isomorphic to $F_{\left\{\pi_{I}\right\}}$ under the mapping $a \rightarrow \sum \mathcal{g}^{*} \bar{\pi}_{I}(a) x^{I}$ where $\left\{\bar{\pi}_{I}\right\}_{g}=\mathcal{E}^{\prime}\left\{\pi_{I}\right\} g$. The correspondence between sequences of derivations on $F$ and embeddings of $F$ in $F\left[\left[x_{1}, \cdots, x_{n}\right]\right]$ is biunique.

\section{BIBLIOGRAPHY}

1. N. Heerema, On ramified complete discrete valuation rings, Proc. Amer. Math. Soc. vol. 10 (1959) pp. 490-496.

2. O. Teichmüller, Über die Struktur diskret bewerteter perfekter Körper, Gott. Nach. vol. 107 (1933) pp. 587-623.

Florida State University 